Artigo original

Hegemonia - Revista Eletrônica do Programa de Mestrado em Direitos Humanos, Cidadania e Violência/Ciência Política do Centro Universitário Unieuro

ISSN: 1809-1261

UNIEURO, Brasília, número 22 (Especial), 2017, pp. 4-26.

Recebido em: 12/7/2017

Avaliado em: 1/8/2017

Aceito em: 23/8/2017

\title{
UMA INVESTIGAÇÃO EMPÍRICA SOBRE A POLÍTICA DE DIVIDENDOS E O RETORNO DE AÇÕES NO BRASIL
}

\author{
Carlos Enrique Carrasco Gutierrez, ${ }^{1}$ Marcello Furlanetto Gomes, ${ }^{2}$ e Tito Belchior Silva Moreira ${ }^{3}$
}

Resumo: Este artigo avalia se a política de distribuição de dividendos afeta o retorno das ações, em empresas brasileiras que possuem ações negociadas na bolsa de valores no período de 2000 a 2009. O modelo base é construído a partir das variáveis retornos das ações, lucros líquidos e dividendos, tendo como variáveis de controle o tamanho da empresa e o endividamento em relação ao preço das ações. Modelos para dados em painel de efeitos fixos e efeitos aleatórios são usados para estimar os impactos dos dividendos nos retornos das ações das firmas. Os resultados baseados nos modelos de efeitos fixos são estatisticamente melhores que os de efeitos aleatórios, e indicam que os dividendos são significantes e afetam positivamente os retornos das ações das firmas. O lucro líquido e o tamanho da firma afetam positivamente, porém não existe evidência que a estrutura de capital afete os retornos das ações. Desta forma, mostrou-se empiricamente através dos resultados econométricos que a geração de lucro e a distribuição de dividendos são indicadores importantes para a valorização de uma empresa de capital aberto no mercado de capitais brasileiro.

Palavras-chave: Dividendos. Retorno. Dados em Painel

Abstract: This paper aims to investigate whether dividend policy affects stock returns in Brazilian companies that have shares traded on the stock exchange in the period 2000 to 2009. The base model is built from the variables: stock returns, dividends and net profit, taking as control variables firm size and debt in relation to stock price. Econometric models for panel data such as fixed effects and random effects models are used to estimate the impact of the dividends on stock returns. The results based on fixed effect models are statistically better than random effects and it indicate that the dividends are significant and positively affect stock returns of firms. Net income and firm size affect positively the stock return but there is no evidence of the capital structure of firms affects the stock returns. Thus, it has been shown empirically through

\footnotetext{
${ }^{1}$ Professor da Pós-Graduação de Economia da Universidade Católica de Brasília.

${ }^{2}$ Mestre em Economia.

${ }^{3}$ Professor da Pós-Graduação de Economia da Universidade Católica de Brasília.
} 
Artigo original

Hegemonia - Revista Eletrônica do Programa de Mestrado em Direitos Humanos, Cidadania e Violência/Ciência Política do Centro Universitário Unieuro

ISSN: 1809-1261

UNIEURO, Brasília, número 22 (Especial), 2017, pp. 4-26.

econometric results that the generation of profit and the distribution of dividends are important indicators for the valuation of a company in the Brazilian capital market.

Keywords: Dividend. Stock Returns. Panel Data.

1. Introdução

As empresas formulam estratégias e ações para aumentar receitas e reduzir seus custos, com o objetivo de maximizar lucro. Para isso devem definir e executar investimentos que podem ser financiados com seus próprios recursos (lucros retidos), por nova emissão de ações (equities) ou por emissão de dívidas (bonds). Segundo Damadoran (1999), as decisões de investimentos podem afetar os fluxos de caixa futuros que determinam o valor de uma empresa. Conhecer o comportamento dessas variáveis pode ser útil aos administradores na adoção de políticas diferenciadas referentes à distribuição de dividendos e na retenção de lucro que possam agregar valor à firma. Pode ser útil também aos investidores para tomadas de decisões de investimento ou desinvestimento em ações.

Existem inúmeros estudos empíricos tentando verificar se os dividendos carregam informações sobre o valor da empresa e o retorno das ações. Diversas teorias surgiram para explicar o comportamento dos preços das ações em função das diferentes políticas de dividendos adotadas pelas empresas. Podemos destacar a teoria da firma de mercado perfeito produzida por Miller e Modigliani (1961) na qual, sobre certas condições de mercado, se estabelece que a política de dividendos é irrelevante para o valor das empresas. Alguns trabalhos empíricos têm tido como ponto de partida esta teoria, entre os mais influentes podemos citar os trabalhos de Black e Scholes $(1973,1974)$, onde os autores encontram evidencias que a teoria dos dividendos (dividend $\left.y_{i e l d}\right)^{4}$ não afeta o retorno das ações. Outros exemplos a citar são Uddin e Chowdhury (2005) e Adesola e Okawong (2009). Por outro lado, temos a teoria de mercado imperfeito de Gordon (1963) e Lintner (1962), a qual pressupõe que o valor da firma é uma função das expectativas futuras do pagamento de dividendos, ou seja, é igual ao valor presente dos infinitos fluxos futuros de dividendos descontados a uma taxa compatível com o mercado e com o risco associado. Tal pressuposto sugere que há uma relação direta entre a política de dividendos da

\footnotetext{
${ }^{4}$ Dividend Yield, abreviadamente DY, é uma expressão inglesa que traduzida literalmente significa rendimento do dividendo. É um índice criado para medir a rentabilidade dos dividendos de uma empresa em relação ao preço de suas ações. Este índice traz o benefício de poder comparar a rentabilidade dos dividendos entre empresas. Obtém-se dividindo o valor dos dividendos recebido no exercício pela cotação da ação no início do exercício.
} 
Artigo original

Hegemonia - Revista Eletrônica do Programa de Mestrado em Direitos Humanos, Cidadania e Violência/Ciência Política do Centro Universitário Unieuro

ISSN: 1809-1261

UNIEURO, Brasília, número 22 (Especial), 2017, pp. 4-26.

empresa e seu valor de mercado. Alguns trabalhos têm corroborado empiricamente esta teoria. Podem ser citados os trabalhos de Campbell e Shiller (1988) e Fama e French (1988) os quais evidenciam que os dividendos são importantes na previsão dos retornos das ações. Podem-se destacar outros relevantes trabalhos sobre o tema, tais como: Milller e Roch (1985); DeAngelo; DeAngelo e Stulz (2006); Denis e Osobov (2008).

Não existem trabalhos conclusivos sobre a matéria já que o tema política de dividendos e seus efeitos sobre o valor da empresa e o retorno de ações é bastante controverso. Tal controvérsia incentiva pesquisadores a explorarem o tema em novas dimensões. Este trabalho tem como objetivo principal investigar se a política de distribuição de dividendos afeta os retornos das ações das empresas brasileiras que possuem ações negociadas na bolsa de valores (BM\&FBovespa) $)^{5}$. Desta forma, este artigo espera contribuir com evidências empíricas a respeito do tema e ampliar o debate sobre os efeitos das políticas de distribuição de dividendos sobre os retorno das ações.

Os resultados empíricos obtidos, neste artigo, fornecem às finanças corporativas algumas contribuições. Inicialmente, fica evidenciada a relação empírica entre a política de dividendos e os retornos das ações das empresas brasileiras. Adicionalmente foram comprovados a influência dos lucros gerados e do tamanho das firmas sobre o retorno das ações. Já a estrutura de capital, nesta pesquisa, representada pelo endividamento de longo prazo, passivo circulante e, inclusive, pelo passivo total, não afeta os retornos das ações.

O restante do artigo está organizado da seguinte forma. Na próxima seção é feita uma breve revisão da literatura sobre artigos internacionais e nacionais relacionados à política de dividendos e ao retorno de ações, destacando-se os resultados obtidos por outros autores. $\mathrm{Na}$ seção três apresenta-se a metodologia utilizada, a descrição e justificativa das variáveis e o modelo proposto de forma a permitir a análise das variáveis lucro e dividendos, como também das variáveis de controle. $\mathrm{Na}$ seção quatro discutem-se os modelos construídos, bem como os resultados empíricos encontrados. Finalmente na seção cinco são relatadas as conclusões.

\footnotetext{
${ }^{5}$ A BM\&FBOVESPA é uma companhia de capital brasileiro formada, em 2008, a partir da integração das operações da Bolsa de Valores de São Paulo e da Bolsa de Mercadorias \& Futuros. Como principal instituição brasileira de intermediação para operações do mercado de capitais, a companhia desenvolve, implanta e provê sistemas para a negociação de ações, derivativos de ações, títulos de renda fixa, títulos públicos federais, derivativos financeiros, moedas à vista e commodities agropecuárias.
} 
Artigo original

Hegemonia - Revista Eletrônica do Programa de Mestrado em Direitos Humanos, Cidadania e Violência/Ciência Política do Centro Universitário Unieuro

ISSN: 1809-1261

UNIEURO, Brasília, número 22 (Especial), 2017, pp. 4-26.

\section{Revisão Bibliográfica}

Diversas teorias $^{6}$ e trabalhos empíricos foram elaborados com o propósito de avaliar os possíveis efeitos da política de dividendos sobre o retorno das ações e o valor da empresa. Pani (2008), por meio de dados em painel, estudou o efeito da política de dividendos sobre os retornos das ações de firmas de diferentes setores que têm ações negociadas na bolsa de valores da India no período de 1996 até 2006. Ele concluiu que a taxa de retenção de dividendos de alguns setores industriais indianos afeta positivamente os retornos das ações e quando a firma aumenta seu endividamento, o valor da firma é afetado negativamente.

Khan et al., (2011) estudaram a relação entre a política de dividendos ${ }^{7}$ e os retornos de ações de uma amostra de 55 empresas listadas no KSE-100 Index com ações na bolsa de valores do Paquistão, no período de 2001 a 2010. Eles concluiram que o dividend yield, lucro por ação, retorno sobre o patrimônio líquido e lucro depois de impostos afetam positivamente os retornos das ações. Por outro lado, mostraram também que a taxa de retenção do lucro líquido tem relação negativa com os retornos das ações das firmas. Asquith e Mullins (1983) investigaram o impacto da distribuição de dividendos sobre a riqueza dos acionistas, através da análise de 168 empresas que têm distribuido dividendos por pelos menos 10 anos. Os resultados empíricos identificaram que subsequentes aumentos dos dividendos podem produzir um maior impacto positivo sobre a riqueza dos acionistas. Rahim et al., (2010) examinaram o impacto do investimento e da governança corporativa sobre a valorização da firma. A amostra contou com 361 empresas não financeiras da Malásia, no período de 2002 a 2007, com dados em painel balançeado. Os resultados obtidos indicaram que os acionistas preferem a relização de investimentos seguros e a manutenção dos níveis de endividamento enquanto distribui o excesso de caixa aos acionistas. Nesse contexto, considera-se que os investidores são avessos ao risco.

No Brasil, alguns trabalhos empíricos tem se dedicado a este assunto. Correia e Amaral (2002) estudaram a relação entre a política de distribuição de resultados de companhias brasileiras sobre o valor de mercado das suas ações. Eles verificaram se o retorno das ações pode ser explicado não apenas pelo risco sistemático, como pressupõe o modelo CAPM, mas também pela

\footnotetext{
${ }^{6}$ Baker, Saadi e Dutta (2011) fazem uma boa revisão sobre as teorias de dividendos. Outros autores também podem ser consultados como Danthine e Donaldson (2005).

7 O Karachi Stock Exchange Index KSE100 compreende a empresa de topo de cada um dos 34 setores na KSE, em termos de capitalização de mercado. O resto das empresas são colhidos no ranking capitalização de mercado, sem qualquer consideração para o setor a fazer uma amostra de 100 ações ordinárias com valor base de 1.000.
} 
Artigo original

Hegemonia - Revista Eletrônica do Programa de Mestrado em Direitos Humanos, Cidadania e Violência/Ciência Política do Centro Universitário Unieuro

ISSN: $1809-1261$

UNIEURO, Brasília, número 22 (Especial), 2017, pp. 4-26.

variável rendimento em dividendos. Os resultados mostraram que tanto o risco sistemático quanto o rendimento em dividendos são fatores que explicam a rentabilidade de mercado das ações. Loss et al., (2003) efetuaram um estudo empírico para verificar a relevância da política de financiamento, em especial, a política de dividendos, sobre três enfoques: a Hipótese da Sinalização, a Hipótese do Agenciamento e a Hipótese da Preferência Tributária e o Efeito Clientela. Eles concluiem que nas três abordagens, ainda não há evidências empíricas conclusivas, especialmente, porque características próprias dos diferentes mercados podem conduzir a administração a estabelecer políticas diferenciadas. Finalmente, Sales (2011) estuda a relação dinâmica entre os lucros contábeis e os retornos acionários das empresas de capital aberto. Os resultados indicam que essa relação é dinâmica e o mercado se antecipa à formação do lucro líquido do exercício, precificando as ações ao longo do ano por meio de ajustes dos preços das ações entre o término do exercício e a divulgação do lucro líquido. Esta pesquisa torna-se relevante dado que são poucos os trabalhos referentes a este tema.

Martins e Famá (2012) fazem uma revisão da literatura sobre política de dividendos no Brasil, com foco nos estudos empíricos realizados no período de 1990 até 2010. Os autores destacam algumas tendências, tais como: relevância da política de dividendos no mercado brasileiro; confirmação da existência de problemas de agência; resultados conflitantes quanto à hipótese da clientela; sinalização e influência dos tributos na definição da política de dividendos; resultados não conflitantes quanto às determinantes da política de dividendos.

Fonteles, et.alli (2013) desenvolvem um estudo com base na Teoria do Pássaro na Mão e na Teoria da Sinalização. O estudo se baseia em Análises de Conteúdo nos Formulários de Referência e nas atas das Assembleias Gerais Ordinárias referentes ao exercício de 2010 e da Análise de Componentes Principais (ACP), para se inferir sobre os possíveis fatores condicionantes de políticas de dividendos elevados. O artigo objetiva caracterizar as empresas integrantes do Índice Dividendos (IDIV) da BM\&FBovespa, averiguando os possíveis fatores condicionantes de políticas de dividendos elevados. A aplicação da ACP possibilitou a identificação de quatro componentes principais: Concentração; Rentabilidade; Prosperidade; e Setor. Constatou-se que a política de dividendos determinada por lei é a mais observada pelas companhias. Conclui-se que, de acordo com a Teoria da Sinalização, as empresas estudadas transmitem sinal positivo ao mercado por meio do planejamento e pagamento de dividendos elevados. 
Artigo original

Hegemonia - Revista Eletrônica do Programa de Mestrado em Direitos Humanos, Cidadania e Violência/Ciência Política do Centro Universitário Unieuro

ISSN: $1809-1261$

UNIEURO, Brasília, número 22 (Especial), 2017, pp. 4-26.

\section{Metodologia}

\subsection{Seleção e coleta de dados}

Os dados foram obtidos da Economática e correspondem ao período de 2000 até 2009 em frequência anual. Partiu-se das informações de todas as empresas transacionadas na Bolsa, cuja quantidade inicial pesquisada supera o número de seiscentas empresas, representada pela BM\&FBovespa. Os critérios de seleção das empresas, no período analisado foram dados pelo histórico consistente de lucro e de pagamento dos dividendos (deve haver ocorrência de lucro no ano e distribuição de dividendo anual) e, segundo, não pode ter havido interrupção no histórico de negociação de ações (o preço anual da ação deve ter variado ao menos uma vez a cada ano analisado).

Obedecendo aos critérios definidos, de uma base de sessenta e nove empresas que obtiveram lucro no período selecionado, restaram 51 empresas, compondo sete setores: Administração de empresas e empreendimentos (3 empresas); Comércio varejista (3 empresas); Empresa de eletricidade, gás e água (6 empresas); Indústria manufatureira (27 empresas); Informação (2 empresas); Mineração (2 empresas) e Serviços financeiros e seguros (8 empresas). As variáveis usadas são obtidas das demonstrações contábeis das empresas e correspondem ao Balanço Patrimonial (BP), a Demonstração do Resultado do Exercício (DRE), a Demonstração das Origens e Aplicações de Recursos (DOAR) e a Demonstração do Fluxo de Caixa (DFC). As contas utilizadas são: Lucro Líquido, Dividendos, inclusive Juros sobre Capital Próprio (JSCP) ${ }^{8}$, Patrimônio Líquido, Reservas de Lucro, Lucros Acumulados, Exigível a Longo Prazo, Passivo Circulante, Passivo Total, Preço de Fechamento, Lucro por Ação. O retorno bruto é calculado pelo preço de fechamento das companhias em relação ao período anterior.

\subsection{Modelo empirico}

Segundo a Hipótese da Eficiência de Mercado (HEM) de Fama (1970), um mercado eficiente é aquele em que as informações disponíveis são refletidas plenamente nos preços

\footnotetext{
8 Os JSCP foram constituídos pela Lei 9.249/95 com o intuito de remunerar o capital dos acionistas ou compensar as perdas ocorridas com a extinção da correção monetária das demonstrações contábeis. Os juros sobre o capital próprio são calculados sobre o patrimônio líquido verificado no ano anterior e são limitados à variação, pro rata dia, da taxa de juros de longo prazo (TJLP).
} 
Artigo original

Hegemonia - Revista Eletrônica do Programa de Mestrado em Direitos Humanos, Cidadania e Violência/Ciência Política do Centro Universitário Unieuro

ISSN: 1809-1261

UNIEURO, Brasília, número 22 (Especial), 2017, pp. 4-26.

correntes e futuros impossibilitando aos investidores qualquer ganho anormal. $\mathrm{O}$ efeito no preço do ativo seria afetado de maneira mais lenta ou mais rápida segundo o conteúdo informacional disponível no mercado. Seguindo a Taxonomia definida por Fama, neste trabalho assume-se o mercado na condição de eficiência semiforte, ou seja, o conjunto de informação inclui também toda a informação publicamente disponível aos participantes do mercado. ${ }^{9}$

Para estabelecer a relação empírica entre a política de dividendos e os retornos das ações das firmas, o uso dos modelos de dados em painel é sugerido. Entre os modelos de painel clássicos temos os modelos de efeitos fixos e efeitos aleatórios que são derivados de uma ordem maior de modelos conhecidos como modelos de efeito específico individual. Estes modelos aproveitam melhor a estrutura em painel que o modelo usual de Pooled.

Considere um modelo de efeito específico individual (individual-specific effects model) com regressões $x_{1} \mathrm{e} x_{2}$ :

$y_{i t}=\alpha_{i}+x_{1 i t} \beta_{1}+x_{2 i t} \beta_{2}+\varepsilon_{i t} \quad i=1, \ldots, N ; t=1, \ldots, T$

$\varepsilon_{i t} \sim i i d\left[0, \sigma_{s}^{2}\right]$

Em que alfa leva em consideração o efeito específico não observável de cada firma, os parâmetros beta 1 e beta2 relacionam as variáveis independentes com a variável dependente. Épsilon é o termo de erro independente e identicamente distribuído com média zero e variância constante.

Este modelo permite que cada unidade transversal tenha intercepto $\alpha_{i}$ diferente enquanto os parâmetros de inclinação são constantes. $\varepsilon_{i t}$ é independente e identicamente distribuido (iid) em $i$ e $t$.

Modelo de Efeitos Fixos

O modelo de efeitos fixo é uma variante do modelo (1) na qual trata $\alpha_{i}$ como uma variável aleatória não observável que captura a heterogeneidade não observável das firmas que se diferenciam em seu tamanho, ativos, dividendos, lucros, etc. Esta variável é potencialmente correlacionada com as variáveis observadas $x_{1}$ e $x_{2}$, o seja, as hipóteses adicionais a ser consideradas são: $\operatorname{corr}\left[\alpha_{i}, x_{1}\right] \neq 0$ e $\operatorname{corr}\left[\alpha_{i}, x_{2}\right] \neq 0$.

\footnotetext{
${ }^{9}$ As empresas estudadas são sociedades anônimas que detêm suas ações negociadas em bolsa de valores.
} 
Artigo original

Hegemonia - Revista Eletrônica do Programa de Mestrado em Direitos Humanos, Cidadania e Violência/Ciência Política do Centro Universitário Unieuro

ISSN: $1809-1261$

UNIEURO, Brasília, número 22 (Especial), 2017, pp. 4-26.

\section{Modelo de Efeitos Aleatórios}

Outra variante do modelo (1) é o modelo de efeitos aleatórios na qual o efeito individual não observável $\alpha_{i}$ é uma variável aleatória distribuída independentemente dos regressores. Neste caso, usualmente se fazem as seguintes hipóteses $\alpha_{i} \sim i d d\left[\alpha, \sigma^{2}\right], \varepsilon_{i t} \sim i d d\left[0, \sigma_{s}^{2}\right], \operatorname{corr}\left[\alpha_{i}, x_{1}\right]=0$ e $\operatorname{corr}\left[\alpha_{i}, x_{2}\right]=0$, não sendo especificado alguma distribuição de probabilidade para $\alpha_{i}$ e $\varepsilon_{i t}$.

Existem diferentes estimadores dos modelos de painel. Neste trabalho usaremos os dois mais citados na literatura o estimador de efeitos fixos e o estimador de efeitos aleatórios.

Estimador de Efeitos Fixos (Within Effects Estimator)

Para estimar o efeito das variáveis $x_{1}$ e $x_{2}$ na variável dependente $y_{i t}$ no modelo (1) os parâmetros $\beta_{1}$ e $\beta_{2}$ devem ser estimados. Isto é feito no modelo transformado (2). A ideia de usar o modelo (2) consiste em anular o efeito específico individual não observável eliminando assim qualquer inconsistencia dos estimadores. O estimador de efeitos fixos dos parâmetros de interesse é nada mais do que o estimador de Mínimos Quadrados Ordinários da equação (2).

$y_{i t}-\bar{y}_{i}=\left(x_{1 i t}-\bar{x}_{1 i}\right) \beta_{1}+\left(x_{2 i t}-\bar{x}_{2 i}\right) \beta_{2}+\varepsilon_{i t}-\bar{\varepsilon}_{i}$

$i=1, \ldots, N ; t=1, \ldots, T$

em que $\bar{y}_{i}=\frac{1}{T} \sum_{t=1}^{T} y_{i t}, \bar{x}_{j i}=\frac{1}{T} \sum_{t=1}^{T} x_{j i t} ;$ para $j=1,2$ e $\bar{\varepsilon}_{i}=\frac{1}{T} \sum_{t=1}^{T} \varepsilon_{i t}$. Esta especificação é obtida substraindo a cada variável do modelo (1) suas respectivas médias amostrais ao longo do tempo. Como $\alpha_{i}=\alpha$ para todo indivíduo $i$, o efeito específico individual é eliminado e em caso exista alguma variável invariante no tempo $x_{i t}=x_{i}$ (exemplo: sexo, raça, etc.) seu efeito em $y_{i t}$ não poderá ser estimado já que esta variável também é eliminada. O estimador de efeitos fixos explora a característica do modelo de painel, para $\mathrm{N}$ grande e $\mathrm{T}$ pequeno fixo (short panel), obtendo um estimador consistente dos parâmetros.

Estimador de Efeitos Aleatórios (Randon Effects Estimator)

O estimador de efeitos aleatórios também explora a característica dos dados em painel. O modelo (1) é transformado em: 
Artigo original

Hegemonia - Revista Eletrônica do Programa de Mestrado em Direitos Humanos, Cidadania e Violência/Ciência Política do Centro Universitário Unieuro

ISSN: $1809-1261$

UNIEURO, Brasília, número 22 (Especial), 2017, pp. 4-26.

$y_{i t}-\hat{\lambda} \bar{y}_{i}=(1-\hat{\lambda}) \mu+\left(x_{1 i t}-\hat{\lambda} \bar{x}_{1 i}\right) \beta_{1}+\left(x_{2 i t}-\hat{\lambda} \bar{x}_{2 i}\right) \beta_{2}+v_{i t}$

$i=1, \ldots, N ; t=1, \ldots, T$

em que $v_{i t}=(1-\hat{\lambda}) \alpha+\left(\varepsilon_{i t}-\hat{\lambda} \bar{\varepsilon}_{1}\right)$ é assintóticamente iid, e $\hat{\lambda}$ um estimador consistente para:

$\lambda=1-\frac{\sigma_{s}}{\sqrt{\sigma_{s}^{2}+T \sigma_{\alpha}^{2}}}$

O estimador de efeitos aleatórios dos parâmetros de interesse é simplesmente o estimador de Mínimos Quadrados Generalizados Factíveis (FGLS) da equação (3).

Os dois modelos tratados acima são os mais usados na literatura e diferem na hipótese sobre a componente específica ou heterogeneidade específica (específicos de cada firma) ser ou não correlacionada com as variáveis independentes. Desta forma, seguindo a equação sugerida por Pani (2008) para a firma $i$ no tempo $t$ (ver apêndice) e considerando $y_{i t}=\ln \left(\frac{P_{\text {it }}}{P_{\text {it }-1}}\right)$, $x_{1 i t}=\ln \left(\frac{L L_{i t}}{P_{i t-1}}\right)$ e $x_{2 i t}=\ln \left(\frac{D_{i t}}{L R_{i t}}\right)$ temos o modelo específico individual:

$\ln \left(\frac{P_{i t}}{P_{i t-1}}\right)=\beta_{0}+\beta_{1} \ln \left(\frac{L L_{i t}}{P_{i t-1}}\right)+\beta_{2} \ln \left(\frac{D_{i t}}{L R_{i t}}\right)+\varepsilon_{i t}$

$\varepsilon_{i t} \sim i i d\left[0, \sigma_{s}^{2}\right]$

$\forall i=1, \ldots, N ; t=1, \ldots, T$

em que; $P_{i t} / P_{i t-1}, L L_{t}$ e $L R_{i t}$ são, respectivamente, o retorno bruto, o Lucro Líquido por ação, e os lucros retidos representados pela soma dos Lucros Acumulados e Reservas de Lucros da firma i no período $t$. $D_{i t}$ representa a parte do lucro líquido da firma $i$ que foi distribuída aos acionistas no tempo $t$ cujo cálculo corresponde ao somatório dos dividendos e Juros sobre Capital Próprio e finalmente $L n$ representa o Logaritmo Neperiano. Definimos as variáveis ${ }^{10}$; $R E T=\ln \left(P_{i t} / P_{\text {itt-1 }}\right), L P A=\ln \left(L L_{i t} / P_{i t-1}\right)$ e $D I L=\ln \left(D_{i t} / L R_{i t}\right)$, em que RET é o retorno das ações, LPA é o lucro por ação dividido pelo preço de fechamento no período anterior e DIL os dividendos em relação aos lucros retidos, e escrevemos, considerando e efeito específico das firmas, a equação [4] como:

\footnotetext{
10 As variáveis explicativas são oriundas de demonstrações financeiras e considera-se que os investidores baseiam suas decisões de compra e venda em expectativas racionais sobre o desempenho da empresa e a conjuntura econômica. Expectativas racionais é a ideia de que os agentes econômicos tentam usar da melhor forma possível as informações de que dispõem para prever o futuro, Simonsen (1986).
} 
Artigo original

Hegemonia - Revista Eletrônica do Programa de Mestrado em Direitos Humanos, Cidadania e Violência/Ciência Política do Centro Universitário Unieuro

ISSN: $1809-1261$

UNIEURO, Brasília, número 22 (Especial), 2017, pp. 4-26.

$$
R E T_{i t}=\alpha_{i}+\beta_{1} L P A_{i t}+\beta_{2} D I L_{i t}+\varepsilon_{i t}
$$

$\varepsilon_{i t} \sim i i d\left[0, \sigma_{s}^{2}\right]$

Para $i=1, \ldots, N ; t=1, \ldots, T$

Em que $\alpha_{i}$ captura o efeito específico individual das firmas. Estudamos outras especificações considerando as seguintes variáveis de controle: ativos totais (ATO); exigível de longo prazo dividido por patrimônio líquido (ELP); passivo circulante dividido por exigível de longo prazo (PCE); passivo total (passivo circulante mais exigível de longo prazo) dividido pelo patrimônio líquido (PTP). As variáveis representadas por ATO, ELP, PCE e PTP, cada uma, correspondem ao logaritmo Neperiano das suas respectivas taxas. As variáveis ELP, PCE e PTP são usadas como indicadores de alavancagem. ELP compara o endividamento de longo prazo, no qual normalmente estão contemplados financiamentos para investimentos da empresa, com o patrimônio líquido. PCE compara o endividamento de curto prazo que, tradicionalmente, tem o endividamento denominado oneroso que é composto de empréstimos bancários, com os financiamentos para investimentos. PTP compara-se toda a dívida da empresa (participação do capital de terceiros) com o patrimônio líquido (capital próprio).

4. Análise dos resultados

$\mathrm{Na}$ Tabela 1, as estimativas do modelo A são mostrados. Este modelo considera como variáveis explicativas a razão do Lucro por Ação sobre o Preço de Fechamento no período anterior (LPA) e a razão dos dividendos sobre os lucros acumulados e reservas de lucros (DIL). O estimador com efeitos fixos (cluster) mostra que todos os coeficientes são significantes ao nível de significância de 1\%. Enquanto que para o modelo com efeitos aleatórios apenas a variável (DIL) é significante ao nível de significância de 10\%.

$\mathrm{O}$ teste $\mathrm{F}$ mostra que existe significância dos parâmetros em conjunto e o $\mathrm{R}^{2}$ um poder explicativo em torno de 0,2. Desta forma este modelo evidencia que as taxas dos lucros e dividendos afetam positivamente os retornos das ações. O modelo B é apresentado, com a inclusão das variáveis de controle ativos totais (ATO), LPA e DIL. Novamente pode ser 
Artigo original

Hegemonia - Revista Eletrônica do Programa de Mestrado em Direitos Humanos, Cidadania e Violência/Ciência Política do Centro Universitário Unieuro

ISSN: $1809-1261$

UNIEURO, Brasília, número 22 (Especial), 2017, pp. 4-26.

observado que o estimador com efeito fixo (cluster) mostra todos os coeficientes significantes ao nível de 5\%. O p-valor da estatística F mostra a existência da significância conjunta das variáveis.

O estimador de efeitos aleatórios mostra a significância apenas para a variável LPA ao nível de 5\%. Na Tabela 2 apresentada no apêndice apresenta-se as estimativas do modelo $\mathrm{C}$ que considera mais uma variável de controle (ELP). Não se identifica a significância da variável Exigível de Longo prazo dividido pelo Patrimônio Líquido (ELP) para os modelos de efeitos fixos e efeitos aleatórios. Em contrapartida as variáveis LPA, DIL e ATO são estatisticamente significantes ao nível de significância de 5\%, para o estimador de efeitos fixos (cluster). Para o estimador de efeitos aleatórios apenas LPA é significante ao nível de 5\%. Finalmente consideramos no modelo D todas as variáveis de controle citadas no texto. O estimador de efeitos fixos (cluster) mostra novamente a significância das variáveis (LPA), (DIL) e (ATO) ao 5\% de nível de significância, porém as variáveis (ELP), (PCE) e (PTP) não são significativas. Para o estimador de efeitos aleatórios as três variáveis representativas do endividamento ELP, PCE e PTP não são significantes, da mesma forma, as variáveis DIL e ATO não são significantes.

Tabela 1: Resultados das Estimações dos modelos de Efeitos Fixos e modelo de Efeitos Aleatórios

\begin{tabular}{cccc}
\hline \multicolumn{4}{c}{$A) R E T_{\text {it }}=\alpha_{\mathrm{i}}+\beta_{1} L P A_{\text {it }}+\beta_{2} D L_{\text {it }}+\varepsilon_{\text {it }}$} \\
\hline \multirow{2}{*}{ Equação } & Efeitos Aleatórios- & Efeitos Fixos & Efeitos Fixos \\
& FGLS (robusto) & (robusto) & $($ cluster $)$ \\
\hline \multirow{2}{*}{ LPA } & $0.19 * * *$ & $0.234 * * *$ & $0.234 * * *$ \\
& $(0.02)$ & $(0.026)$ & $(0.019)$ \\
& {$[0.000]$} & {$[0.000]$} & {$[0.000]$} \\
DIL & 0.021 & 0.047 & $0.047 * * *$ \\
& $(0.012)$ & $(0.024)$ & $(0.011)$ \\
& {$[0.098]$} & {$[0.053]$} & {$[0.006]$} \\
Constante & $0.597 * * *$ & $0.707 * * *$ & $0.707 * * *$ \\
& $(0.043)$ & $(0.06)$ & $(0.045)$ \\
& {$[0.000]$} & {$[0.000]$} & {$[0.0000]$} \\
\hline Prob $>$ F & & 0 & 0
\end{tabular}


Artigo original

Hegemonia - Revista Eletrônica do Programa de Mestrado em Direitos Humanos, Cidadania e Violência/Ciência Política do Centro Universitário Unieuro

ISSN: $1809-1261$

UNIEURO, Brasília, número 22 (Especial), 2017, pp. 4-26.

\begin{tabular}{cccc}
\hline Prob $>$ chi2 & 0 & - & - \\
\hline R2 & 0.194 & 0.192 & 0.192 \\
\hline Observações & 459 & 459 & 459 \\
\hline
\end{tabular}

B) $R E T_{\text {it }}=\alpha_{i}+\beta_{1} L P A_{\text {it }}+\beta_{2} D I L_{\text {it }}+\beta_{2} A T O_{\text {it }}+\varepsilon_{\text {it }}$

\begin{tabular}{|c|c|c|c|}
\hline Equação & $\begin{array}{c}\text { Efeitos Aleatórios } \\
\text { (robusto) }\end{array}$ & $\begin{array}{c}\text { Efeitos Fixos } \\
\text { (robusto) }\end{array}$ & $\begin{array}{c}\text { Efeitos Fixos } \\
\text { (cluster) }\end{array}$ \\
\hline \multirow{3}{*}{ LPA } & $0.194 * * *$ & $0.268^{* * *}$ & $0.268^{* * *}$ \\
\hline & $(0.02)$ & $(0.037)$ & $(0.03)$ \\
\hline & {$[0.000]$} & {$[0.000]$} & {$[0.000]$} \\
\hline \multirow{3}{*}{ DIL } & 0.02 & $0.048^{* *}$ & $0.048^{* * *}$ \\
\hline & $(0.013)$ & (0.024) & $(0.012)$ \\
\hline & [0.118] & {$[0.047]$} & {$[0.006]$} \\
\hline \multirow{3}{*}{ ATO } & 0.006 & $0.123^{* *}$ & $0.123^{* *}$ \\
\hline & (0.004) & $(0.049)$ & $(0.036)$ \\
\hline & {$[0.18]$} & {$[0.015]$} & {$[0.014]$} \\
\hline \multirow{3}{*}{ Constante } & $0.516^{* * *}$ & -1.064 & - 1.064*** \\
\hline & $(0.085)$ & $(0.672)$ & $(0.48)$ \\
\hline & {$[0.000]$} & {$[0.12]$} & {$[0.0000]$} \\
\hline Prob $>$ F & & 0 & 0 \\
\hline Prob > chi2 & 0 & - & - \\
\hline $\mathrm{R} 2$ & 0.192 & 0.083 & 0.083 \\
\hline Observações & 459 & 459 & 459 \\
\hline
\end{tabular}

Notas: *** indica significância estatística a $1 \%$. ** indica significância estatística a $5 \%$. * indica significância estatística a 10\%. Erros-padrão entre parênteses e p-valor entre colchetes. O estimador robusto por cluster foi selecionado agrupando as firmas por setores. RET é o retorno das ações, LPA é o lucro por ação dividido pelo preço de fechamento no período anterior e DIL os dividendos em relação aos lucros retidos. Modelo de efeitos aleatórios estimado pelo Método FGLS (Feasible General Least Squares).

Em resumo, a taxa de dividendos (DIL) que representa quanto é distribuído em relação ao lucro gerado e acumulado pela companhia, evidencia a relação positiva desta variável com o 
Artigo original

Hegemonia - Revista Eletrônica do Programa de Mestrado em Direitos Humanos, Cidadania e Violência/Ciência Política do Centro Universitário Unieuro

ISSN: 1809-1261

UNIEURO, Brasília, número 22 (Especial), 2017, pp. 4-26.

retorno da ação, resultado similar encontrado em Pani (2008). Em relação ao indicador taxa do lucro líquido (LPA), que representa a taxa que o lucro gerado por ação remunera ou rentabiliza o valor pago por ela, evidencia a relação positiva desta variável e os retornos das firmas. Este resultado está em linha com o encontrado por Khan et al. (2011). Por tanto, é possível estabelecer uma relação causal entre as variáveis DIL e LPA e os retornos das firmas (RET). Em relação às variáveis de controle correspondentes a três representações do endividamento de uma empresa, que representa o grau de alavancagem da empresa, não apresentam informação para a explicação do retorno das firmas.

A Tabela 3 (apêndice) mostra as estatísticas descritivas e a matriz de correlação dos indicadores construídos. Esta matriz mostra que em geral, os valores de correlação entre as variáveis estudadas são baixos o que permite considerar todas as variáveis em diversas especificações a serem estudadas.

A hipótese de modelos de efeitos fixos e efeitos aleatórios são considerados na especificação (5) e estimados por meio dos estimadores efeitos fixos (Within estimator) e efeitos aleatórios (random effects estimator). Estes dois estimadores são adequados quando a especificação assumida do modelo para dados em painel (Processo Gerador de Dados - DGP) correspondem, respectivamente, a um modelo de efeitos fixos e modelo de efeitos aleatórios.

A escolha da melhor especificação entre os modelos de efeitos fixos ou de efeitos aleatórios é feita usando o teste de Hausman. Nas estimativas a seguir o teste indicou, em todos os casos, os modelos com efeitos fixos mais apropriados, consequentemente os estimadores de efeitos fixos são usados por serem os mais apropriados para esta especificação. Vejamos o resultado do teste de Hausman no modelo A na tabela 1. O teste forneceu um p-valor de 0,0025 indicando a rejeição da hipótese nula o que favorece a escolha do modelo de efeitos fixos ${ }^{11}$. No entanto, devido a críticas de baixo poder do teste de Hausman consideramos em todos os casos os modelos de efeitos aleatórios e suas estimativas usando o estimador de efeitos aleatórios. Os resultados são mostrados nas Tabelas 1 e 2.

Foram aplicados os testes de heterocedasticidade de Breusch-Pagan/LM para os modelos de efeitos aleatórios e de Wald Modificado para os modelos de efeitos fixos. Ambos os testes

\footnotetext{
11 A aceitação da Hipótese nula do teste de Hausman implica na escolha do modelo de Efeitos Aleatórios, ao passo que a rejeição da hipótese nula indica a escolha do modelo de efeitos fixos. Para os modelos B, C e D os valores do p-valor foram, respectivamente, de 0,0007, 0,0024 e 0,0113 rejeitando em todos os casos a hipótese nula.
} 
Artigo original

Hegemonia - Revista Eletrônica do Programa de Mestrado em Direitos Humanos, Cidadania e Violência/Ciência Política do Centro Universitário Unieuro

ISSN: $1809-1261$

UNIEURO, Brasília, número 22 (Especial), 2017, pp. 4-26.

identificaram heterocedasticidade para as diversas especificações. Desta forma, corrigiu-se o problema usando o estimador de efeitos fixos e efeitos aleatórios robustos a heterocedasticidade e autocorrelação.

\section{Conclusões}

Esse artigo tem como objetivo principal investigar a relação entre o pagamento de dividendos e o retorno das ações, utilizando uma amostra de 51 empresas com ações negociadas na bolsa de valores brasileira (BM\&Fbovespa), no período de 2000 a 2009. A abordagem com dados em painel com método de estimação de efeitos fixos apresentou estimadores mais robustos do que com efeitos aleatórios, conforme mostrados nas Tabelas 1 e 2. Os resultados empíricos obtidos evidenciam a relação empírica positiva entre a política de dividendos e os retornos das ações das empresas brasileiras com ações na bolsa. Adicionalmente foram comprovados a influencia dos lucros gerados no retorno das ações.

Com relação à variável de controle ativo total que representa o tamanho da firma, conclui-se que afeta positivamente os retornos das ações. Esse comportamento não pode ser amparado pelo "efeito tamanho" apresentado por Banz (1981), pois o autor achou uma relação negativa entre tamanho da firma e retorno das ações. Entretanto, Perdigão (1983:150) chegou em seu relevante trabalho: "( ... ) esses conglomerados (os maiores) mostraram, também, maior capacidade de controlar custos e obter lucro, o que, provavelmente, resulta da realização de economias de escala ou economias de diversificação". Em outras palavras, o autor achou uma relação positiva entre retorno e tamanho das firmas.

Com relação às variáveis de controle que representam a alavancagem das empresas, apesar da utilização de três representações do endividamento de uma empresa, não houve evidencias de relação significativa com os retornos das ações, mostrando que a estrutura de capital, não afeta os retornos das ações das firmas.

Desta forma, mostrou-se empiricamente através dos resultados econométricos que a geração de lucro e a distribuição de dividendos são indicadores importantes para a valorização de uma empresa de capital aberto no mercado de capitais brasileiro e representativo para uma economia emergente. 
Artigo original

Hegemonia - Revista Eletrônica do Programa de Mestrado em Direitos Humanos, Cidadania e Violência/Ciência Política do Centro Universitário Unieuro

ISSN: 1809-1261

UNIEURO, Brasília, número 22 (Especial), 2017, pp. 4-26.

\section{Referências}

ADESOLA, W. A., e OKWONG, A. E. An Emparical Study of Dividend Policy of Quoted Companies in Nigeria. Global Journal of Social Sciences, 8 (1), 85-101, 2009.

ASQUITH, P., MULLINS, D.W.Jr, (1983). The Impact of Initiating Dividend Payments on Shareholders' Wealth. The Journal of Business, Vol. 56, No. 1 pp. 77-96, Jan. 1983.

BALTAGI, B.H, Econometric Analysis of Panel Data. Ed. John Wiley \& Sons, Ltd. 2005.

BANZ, R. W. The relationship between return and market value of common stock. Journal of Financial Economics, 9, p. 3-18. 1981.

BAKER, H. K.; SAADI, S.; DUTTA, S. e GANDHI, D. (2011). The perception of dividends by Canadian managers: new survey evidence. International Journal of Managerial Finance, V. 3, N.1.

BLACK, SCHOLES. The princing of options and corporate liabilities. Journal of Political Economy, 4(81), 637-654. 1973.

. The effects of dividend yield and dividend policy on common stock prices and returns. Journal of Financial Economics, Volume 1, Issue 1, Pages 1-22, 1974.

CAMPBELL, J Y, SHILLER, R. J. The dividend-price ratio and expectations of future dividends and discount factors. The Review of Financial Studies, Vol. 1, Issue 3, Pp. 195-228, 1998.

CAMPBELL, J Y, SHILLER, R. J. Valuation Ratios and The Long-Run Stock Market Outlook. The Journal of Portfolio Management, 1998.

CHEOLBEOM PARK. When does dividend-price ratio predict stock returns? Journal of Empirical Finance, 17 (2010) 81-101.

CORREIA L, FERRAZ, E AMARAL H.F. O Impacto da Política de Dividendos sobre a Rentabilidade de Títulos Negociados na Bovespa no Período de 1994 a 2000. Associação Nacional de Pós-Graduação e Pesquisa em Administração, 2002. 
Artigo original

Hegemonia - Revista Eletrônica do Programa de Mestrado em Direitos Humanos, Cidadania e Violência/Ciência Política do Centro Universitário Unieuro

ISSN: 1809-1261

UNIEURO, Brasília, número 22 (Especial), 2017, pp. 4-26.

CUTHBERTSON, KEITH E NITZSCHE D, Quantitative Financial Economics. Second Edition, Ed. John Wiley \& Sons, Ltd, 2004.

DANTHINE, JEAN-PIERRE e DONALSON, J. B. Intermediate Financial Theory. Academic Press, 2nd Edition, 2005.

DeANGELO, H.; LINDA DeANGELO e RENÉ M. STULZ, Dividend policy and the earned/contributed capital mix: a test of the life-cycle theory. Journal of Financial Economics, Vol. 81, n. 2, PP: 227-254, August, 2006.

DENIS, DAVID J. e IGOR OSOBOV. Why do firms pay dividends? International evidence on the determinants of dividend policy. Journal of Financial Economics, Vol. 89, n. 1, PP: 62-82, July. 2008.

FAMA, E.F, FRENCH, K.R, Dividend Yields and Expected Stock Returns. Journal of Financial Economics, Volume 22, Issue 1, Pages 3-25, 1988.

FAMA, E.F, FRENCH, K.R,. Efficiente Capital Markets: a review of theory and empirical work. Journal of Financial Economics, vol. 25(2), dec. 1970.

FONTELES, ISLANE V; PEIXOTO, JR, C. A.; VASCONCELOS, A. C.; DE LUCA, M. M. MENDES. (2012). Política de Dividendos das Empresas Participantes do Índice Dividendos da Bm\&FBovespa. Revista Contabilidade Vista \& Revista, Universidade Federal de Minas Gerais, Belo Horizonte, v. 23, n. 3, p. 173 -204, jul./set.

GREENE, W.H, Econometric Analysis, Ed. Pearson Prentice Hall, 2008.

KHAN et al. Can Dividend Decisions Affect the Stock Prices: A Case of Dividend Paying Companies of KSE. International Research Journal of Finance and Economics, 2011.

LOSS, LENITA, NETO A.S Política de Dividendos, na prática, É importante? Revista Contabilidade \& Finanças - USP, São Paulo, 2003.

MARTINS, ANDRESSA L. (2012). O que revelam os estudos realizados no Brasil sobre políticas de dividendos? RAE, São Paulo, v. 52, n. 1, jan/ fev., p. 024-039 
Artigo original

Hegemonia - Revista Eletrônica do Programa de Mestrado em Direitos Humanos, Cidadania e Violência/Ciência Política do Centro Universitário Unieuro

ISSN: $1809-1261$

UNIEURO, Brasília, número 22 (Especial), 2017, pp. 4-26.

MILLER, MERTON, MODIGLIANI F, Dividend Policy, Growth, and Valuation of Shares. Journal of Business. 34. PP. 411-433, 1961.

MILLLER, MERTON E KEVIN ROCH, Dividend Policy under Asymmetric Information. The Journal of Finance, Vol. 40, N. 4, PP. 1031-1051, September, 1985.

PANI. U, Dividend Policy and Stock Price Behaviour in Indian Corporate Sector: A panel data approach. Retrieved from Indian Institute of Technology, 2008.

PERDIGÃO, LUIZ ANTÔNIO. Conglomerados financeiros: análise de seu desempenho no Brasil (1978/1981). Rio de Janeiro, Instituto Brasileiro de Mercado de Capitais, 1983

RAHIM, RUZITA A., YAACOB M.H, ALIAS N, NOR, FAUZIAAS M.N. Investment, Board Governance and Firm Value: A Panel Data Analysis. International Review of Business Research Papers, Volume 6. Number 5. Pp. 293 - 302. Nov 2010.

SALES. I.C.H A Dinâmica da Relação entre os Lucros Contábeis e os Retornos acionários nas empresas brasileiras de capital aberto. Dissertação de Mestrado, UNB, 2011.

UDDIN M. H., CHOWDHURY, G. M. Effects of Dividend Announcement on Shareholders' Value: Evidence from Dhaka Stock Exchange. Journal of Business Research, 7, 61-72. (2005). 
Artigo original

Hegemonia - Revista Eletrônica do Programa de Mestrado em Direitos Humanos, Cidadania e Violência/Ciência Política do Centro Universitário Unieuro

ISSN: $1809-1261$

UNIEURO, Brasília, número 22 (Especial), 2017, pp. 4-26.

Apêndice A: Resultados e Estatísticas Descritivas

Tabela 2: Resultados das Estimações dos modelos de Efeitos Fixos e modelo de Efeitos Aleatórios

\begin{tabular}{|c|c|c|c|}
\hline Equação & $\begin{array}{l}\text { Efeitos Aleatórios } \\
\text { (robusto) }\end{array}$ & $\begin{array}{c}\text { Efeitos Fixos } \\
\text { (robusto) }\end{array}$ & $\begin{array}{c}\text { Efeitos Fixos } \\
\text { (cluster) }\end{array}$ \\
\hline \multirow{3}{*}{ LPA } & $0.195^{* * *}$ & $0.268^{* * *}$ & $0.268^{* * *}$ \\
\hline & $(0.02)$ & $(0.037)$ & $(0.03)$ \\
\hline & [0.000] & [0.000] & [0.000] \\
\hline \multirow{3}{*}{ DIL } & 0.018 & $0.047^{*}$ & $0.047 * * *$ \\
\hline & $(0.012)$ & (0.024) & (0.011) \\
\hline & [0.153] & {$[0.054]$} & [0.005] \\
\hline \multirow{3}{*}{ ATO } & $0.012^{*}$ & $0.127 * *$ & $0.127 * *$ \\
\hline & $(0.007)$ & $(0.05)$ & $(0.041)$ \\
\hline & [0.091] & [0.015] & [0.021] \\
\hline \multirow{3}{*}{ ELP } & -0.014 & -0.009 & -0.009 \\
\hline & $(0.009)$ & $(0.019)$ & $(0.013)$ \\
\hline & [0.123] & [0.642] & [0.518] \\
\hline \multirow{3}{*}{ Constante } & $0.409 * * *$ & -1.12 & $0.563^{*}$ \\
\hline & $(0.133)$ & (0.699) & $(0.045)$ \\
\hline & [0.002] & [0.115] & [0.094] \\
\hline Prob $>$ F & & 0 & 0 \\
\hline Prob > chi2 & 0 & - & - \\
\hline $\mathrm{R} 2$ & 0.198 & 0.0846 & 0.0846 \\
\hline Observações & 459 & 459 & 459 \\
\hline
\end{tabular}

\begin{tabular}{cccc}
\hline Equação & $\begin{array}{c}\text { Efeitos Aleatórios } \\
\text { (robusto) }\end{array}$ & $\begin{array}{c}\text { Efeitos Fixos } \\
\text { (robusto) }\end{array}$ & $\begin{array}{c}\text { Efeitos Fixos } \\
\text { (cluster) }\end{array}$ \\
\hline LPA & $0.195^{* * *}$ & $0.268^{* * *}$ & $0.268^{* * *}$
\end{tabular}


Artigo original

Hegemonia - Revista Eletrônica do Programa de Mestrado em Direitos Humanos, Cidadania e Violência/Ciência Política do Centro Universitário Unieuro

ISSN: 1809-1261

UNIEURO, Brasília, número 22 (Especial), 2017, pp. 4-26.

\begin{tabular}{|c|c|c|c|}
\hline & $(0.02)$ & $(0.037)$ & 0.03 \\
\hline & {$[0.000]$} & {$[0.000]$} & {$[0.000]$} \\
\hline & 0.017 & $0.047^{*}$ & $0.047 * * *$ \\
\hline \multirow[t]{3}{*}{ DIL } & $(0.013)$ & $(0.024)$ & 0.011 \\
\hline & {$[0.17]$} & {$[0.056]$} & {$[0.006]$} \\
\hline & 0.014 & $0.134 * *$ & $0.134 * *$ \\
\hline \multirow[t]{3}{*}{ ATO } & $(0.009)$ & $(0.052)$ & 0.043 \\
\hline & {$[0.115]$} & {$[0.014]$} & {$[0.021]$} \\
\hline & 0.039 & 0.064 & 0.064 \\
\hline \multirow[t]{3}{*}{ ELP } & $(0.1)$ & $(0.136)$ & 0.126 \\
\hline & {$[0.697]$} & {$[0.642]$} & {$[0.631]$} \\
\hline & 0.042 & 0.063 & 0.063 \\
\hline \multirow[t]{3}{*}{ PCE } & $(0.076)$ & $(0.108)$ & $(0.102)$ \\
\hline & {$[0.58]$} & {$[0.566]$} & {$[0.561]$} \\
\hline & -0.055 & -0.072 & -0.072 \\
\hline \multirow[t]{3}{*}{ PTP } & $(0.109)$ & $(0.158)$ & $(0.142)$ \\
\hline & {$[0.618]$} & [0.649] & [0.629] \\
\hline & $0.42 * * *$ & $-1.183^{*}$ & $-1.183^{*}$ \\
\hline \multirow[t]{2}{*}{ Constante } & $(0.138)$ & $(0.703)$ & $(0.581)$ \\
\hline & {$[0.002]$} & [0.098] & [0.088] \\
\hline Prob $>$ F & & 0 & 0 \\
\hline Prob $>$ chi 2 & 0 & - & - \\
\hline $\mathrm{R} 2$ & 0.198 & 0.082 & 0.08 \\
\hline Observações & 459 & 459 & 459 \\
\hline
\end{tabular}

Notas: A variável dependente é RET. *** indica significância estatística a 1\%. ** indica significância estatística a 5\%. * indica significância estatística a 10\%. Erros-padrão entre parênteses e p-valor entre colchetes. O estimador robusto por cluster foi selecionado agrupando as firmas por setores. RET é o retorno das ações, LPA é o lucro por ação dividido pelo preço de fechamento no período anterior e DIL os dividendos em relação aos lucros retidos. 
Artigo original

Hegemonia - Revista Eletrônica do Programa de Mestrado em Direitos Humanos, Cidadania e Violência/Ciência Política do Centro Universitário Unieuro

ISSN: 1809-1261

UNIEURO, Brasília, número 22 (Especial), 2017, pp. 4-26.

Tabela 3: Estatísticas Descritivas Básicas

\begin{tabular}{lcccc}
\hline & Média & Máximo & Mínimo & Desvio- padrão \\
RET & 0.257 & 1.435 & -1.340 & 0.424 \\
LPA & -1.629 & 0.937 & -5.158 & 0.957 \\
DIL & -1.460 & 3.041 & -4.515 & 1.253 \\
ATO & 14.807 & 20.379 & 10.549 & 2.262 \\
ELP & -1.022 & 2.663 & -13.580 & 1.799 \\
PCE & 0.782 & 7.712 & -1.726 & 1.216 \\
PTP & 0.266 & 3.572 & -5.868 & 1.271 \\
& & & & \\
\hline
\end{tabular}


Artigo original

Hegemonia - Revista Eletrônica do Programa de Mestrado em Direitos Humanos, Cidadania e Violência/Ciência Política do Centro Universitário Unieuro

ISSN: 1809-1261

UNIEURO, Brasília, número 22 (Especial), 2017, pp. 4-26.

Tabela 4: Matriz de Correlação para as variáveis de interesse

\begin{tabular}{lccccccc}
\hline & RET & LPA & DIL & ATO & ELP & PCE & PTP \\
RET & 1.000 & & & & & & \\
LPA & 0.437 & 1.000 & & & & & \\
DIL & 0.112 & 0.118 & 1.000 & & & & \\
ATO & -0.067 & -0.233 & 0.076 & 1.000 & & & \\
ELP & -0.065 & -0.088 & -0.051 & 0.547 & 1.000 & & \\
PCE & 0.058 & 0.079 & -0.030 & -0.404 & -0.699 & 1.000 & \\
PTP & -0.052 & -0.071 & -0.091 & 0.505 & 0.885 & -0.2998 & 1.000 \\
& & & & & & & \\
\hline
\end{tabular}

Fonte: Elaboração própria. 
Artigo original

Hegemonia - Revista Eletrônica do Programa de Mestrado em Direitos Humanos, Cidadania e Violência/Ciência Política do Centro Universitário Unieuro

ISSN: $1809-1261$

UNIEURO, Brasília, número 22 (Especial), 2017, pp. 4-26.

Apêndice B: Modelo de Pani (2008)

Pani (2008) em seu artigo que estuda as corporações indianas propõe um modelo a ser testado. Partindo da hipótese que o valor da firma é afetado pela política de dividendos o modelo sugerido por Pani é escrito como:

$\frac{P_{t}}{P_{t-1}}=f\left(\frac{L L_{t}}{P_{t-1}}, \frac{D_{t}}{L R_{t}}\right)$

em que $P_{t} / P_{t-1}$ é o retorno da firma, $L L_{t}$ é o Lucro Líquido da empresa, $D_{t}$ representa a parte do lucro líquido que foi distribuída aos acionistas cujo cálculo corresponde ao somatório dos dividendos e Juros sobre Capital Próprio e $L R_{t}$ são os lucros retidos representados pela soma dos Lucros Acumulados e Reservas de Lucros. É considerada uma visão simplificada da função $f($.$) como sendo uma função Cobb-Douglas medida pelo retorno bruto, e como inputs os ratios$ lucro líquido em relação ao preço anterior $\left(L L_{t} / P_{t-1}\right)$ e dos dividendos em relação aos lucros retidos $\left(D_{t} / L R_{t}\right)$.

$\frac{P_{t}}{P_{t-1}}=A\left(\frac{L L_{t}}{P_{t-1}}\right)^{\beta_{1}}\left(\frac{D_{t}}{L R_{t}}\right)^{\beta_{2}} e^{u_{\tilde{i}}}$

em que $A$ é um fator de produtividade da firma, $\beta_{1}$ e $\beta_{2}$ são, respectivamente, as elasticidades do lucro líquido em relação ao preço anterior e dos dividendos em relação aos lucros retidos. Considerando a propriedade de monotonicidade da função de Cobb-Douglas da Equação [4], podemos expressar esta equação em função de seu logaritmo Neperiano $\ln ($.$) , para a firma i$ no tempo $t$ como:

$\ln \left(\frac{P_{i t}}{P_{i t-1}}\right)=\ln (A)+\beta_{1} \ln \left(\frac{L L_{i t}}{P_{i t-1}}\right)+\beta_{2} \ln \left(\frac{D_{i t}}{L R_{i t}}\right)+u_{i t}$

$\varepsilon_{i t} \sim i i d\left[0, \sigma_{s}^{2}\right]$

$\forall i=1, \ldots, N ; \quad t=1, \ldots, T$ 